\title{
Suppression of stored-beam oscillation and observation of flux improvement during top-up injection
}

\author{
Y-G. Son, ${ }^{1}$ J.-Y. Kim, ${ }^{1}$ C. Mitsuda,${ }^{2}$ K. Kobayashi, ${ }^{2}$ J. Ko, ${ }^{1}$ T-Y. Lee, ${ }^{1}$ J-Y. Choi, ${ }^{1}$ D-E. Kim, ${ }^{1}$ \\ H-S. Seo, ${ }^{1}$ H-S. Han, ${ }^{1}$ K-S. Park, ${ }^{1}$ C-D. Park, ${ }^{1}$ and S. Shin ${ }^{1, *}$ \\ ${ }^{1}$ Pohang Accelerator Laboratory, POSTECH, Pohang, Kyungbuk 790-784, Korea \\ ${ }^{2}$ SPring-8, Kouto, Sayo-cho, Sayo-gun, Hyogo 679-5198, Japan
}

(Received 9 August 2016; published 30 August 2017)

\begin{abstract}
This paper describes a start-to-end study of the suppression of stored-beam oscillation during top-up injection at the Pohang Light Source (PLS-II) beam line. The fast counterkicker implemented in PLS-II suppresses stored-beam oscillations in the vertical plane. During top-up injection in the magnetic spectroscopy beam line of PLS-II, flux improvement due to the suppression of stored-beam oscillation was clearly observed.
\end{abstract}

DOI: 10.1103/PhysRevAccelBeams.20.082803

\section{INTRODUCTION}

As a result of successes at Advanced Photon Source (APS) [1] and Swiss Light Source [2], top-up operation is becoming the standard mode of operation in thirdgeneration light sources. Top-up operation has achieved a constant beam current in the storage ring to overcome limitations to the lifetime of the electron beam and to obtain a constant photon flux for a thermal equilibrium at the beam line. Pohang Light Source (PLS-II) [3] has also been successfully performing top-up operation since March 2013. At the supramolecular crystallography beam line of PLS-II, the electron beam current has been regulated within $0.1 \%$ rms deviation at $400 \mathrm{~mA}$, and the flux change for $8 \mathrm{~h}$ has reduced from $40 \%$ in the decay mode to $2 \%$ in top-up operation [4].

During top-up operation, the beam-injection system excites an oscillation of the stored beam. The excited oscillation effectively increases stored-beam emittance and modulates the photon-beam intensity. Furthermore, future light sources based on a storage ring will have ultralow emittance that requires an extremely stable beam. In these light sources, the lifetime of a stored beam will be extremely short, and the frequency of beam injection for top-up will increase. Therefore, to achieve constant photonbeam intensity during top-up operation for user experiments in the future low-emittance ring, the stored-beam oscillation must be suppressed during top-up injection.

In general, stored-beam oscillation during injection is caused by an imperfection of the injection system, such as the tilt of bump magnets and the dissimilarity of their

\footnotetext{
*tlssh@postech.ac.kr
}

Published by the American Physical Society under the terms of the Creative Commons Attribution 4.0 International license. Further distribution of this work must maintain attribution to the author(s) and the published article's title, journal citation, and DOI. magnetic fields. The oscillations can also be caused by the inherent characteristic on each injection system: e.g., nonlinearity within an injection bump orbit and the leakage field from septum magnets. One method to suppress the stored-beam oscillation is to reduce the imperfection of the injection system; another is to implement a subsystem to compensate for this imperfection.

A fast counterkicker as a method to suppress the storedbeam oscillation has been developed and tested in PLS-II and in SPring-8. In this paper, we describe the stored-beam oscillation, the result of the suppression of the stored-beam oscillation by the fast counterkicker, and the observation of flux improvement during top-up injection. Section II introduces the PLS-II injection system. Section III introduces the theoretical description of stored-beam oscillation during topup injection. Section IV describes the development of a fast counterkicker. Section V describes the experimental result of the oscillation suppression and the flux improvement by the fast counterkicker. Section VI presents the conclusions.

\section{PLS-II INJECTION SYSTEM}

Four bump magnets for an orbit bump are located within the 6.8-m-long straight section in PLS-II. The bump magnet is operated at a $10-\mathrm{Hz}$ repetition rate and has a $7-\mu$ s half-sine wave, so the rise and fall times are both $3.5 \mu \mathrm{s}$. The incoming electron beam from the beam transfer line is horizontally parallel to the bumped orbit in the storage ring and is injected $8.234^{\circ}$ vertically. A Lambertson magnet then bends the beam $-8.234^{\circ}$ vertically to place the incoming electron beam on the same plane as the bumped orbit. In PLS-II, the main source of the horizontal beam oscillation is nonsimilarity of the bump magnets, and the main source of the vertical beam oscillations is the leakage field of the Lambertson septum magnet. As a result, 1\%$8 \%$ systematic dips in the photon beam flux pattern of each beam line occur during beam injection in PLS-II [4]. 
The Lambertson septum magnet [Figs. 1(a) and 1(b)] [5] for PLS-II generates an integrated leakage field [Fig. 1(c)] along the bump height from a field measurement. The integrated leakage field is less than the $1000 \mathrm{G} \mathrm{cm}$ tolerance specification, and the difference between the integrated leakage fields at the stored beam's position and the bumped orbit's position is $230 \mathrm{G} \mathrm{cm}$ [Fig. 1(c)]. The integrated leakage field decreases linearly with decreases in the magnitude of the bump height; this relationship implies that the vertical oscillation caused by the leakage field may be easily suppressed by imposing a counteracting linear source. Various restrictions limit the amount by which the leakage field can be reduced. Therefore, rather than removing the oscillation source, a desirable strategy is to develop a method

(a)

(b)
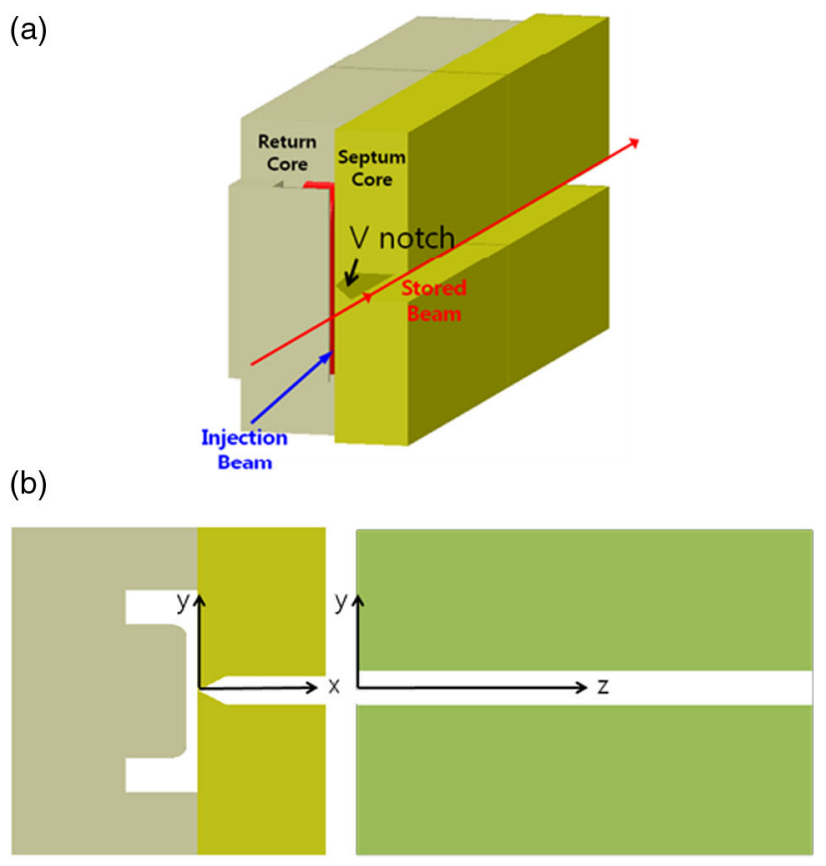

(c)

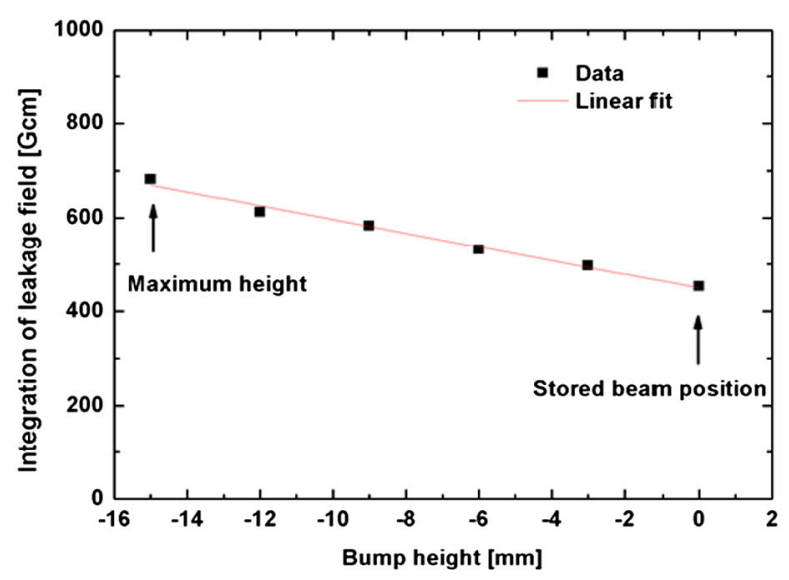

FIG. 1. (a) Model of the Lambertson septum magnet. (b) Definition of coordinates along the septum magnet. (c) Integrated leakage field along the bump height ( $x$ axis). to compensate for the error that causes the stored-beam oscillation by the Lambertson septum magnet.

Previous studies at PLS-II [5] considered alternative methods to suppress the vertical stored-beam oscillation caused by the Lambertson septum: A field compensation magnet around the septum magnet (i.e., a skew-bending magnet and a skew-quadrupole magnet) can suppress the oscillation of the stored beam caused by the leakage field; by tilting the bumped orbit at the kicker deliberately, the vertical kick from the kicker can be used to compensate for the effect of the septum's leakage field; and also the adjustment of the vertical betatron tune can reduce the vertical oscillation induced by the septum magnet. However, the applicability of all these methods is limited by side effects such as the restricted space for installation, the effort required for successful implementation, and undesirable changes of the beam parameters. Therefore, in spite of the aforementioned methods, there still remained the need for a simple and straightforward technique to suppress the vertical beam oscillation due to the Lambertson septum magnet.

\section{BEAM OSCILLATION DURING INJECTION}

To describe the perturbation caused by the imperfection of the injection system, we can use a one-turn transfer matrix $T$ to track the particle motion [6]:

$$
\left(\begin{array}{l}
x \\
x^{\prime} \\
y \\
y^{\prime}
\end{array}\right)_{n}=T\left(\begin{array}{c}
x \\
x^{\prime}+\theta_{x} \\
y \\
y^{\prime}+\theta_{y}
\end{array}\right)_{n-1},
$$

where $n$ is the index for the turn number and $\theta$ represents the kick induced by the imperfection of the injection system. Here the full-turn coupled transfer matrix $T$ is decomposed into normal modes as

$$
T=\left(\begin{array}{cc}
M & n \\
m & N
\end{array}\right)=V U V^{-1}
$$

where

$$
\begin{aligned}
& U=\left(\begin{array}{ll}
A & 0 \\
0 & B
\end{array}\right) ; \quad V=\left(\begin{array}{cc}
\gamma I & C \\
-C^{\dagger} & \gamma I
\end{array}\right) \text { and } \gamma^{2}+|C|=1 . \\
& A=\left(\begin{array}{cc}
\cos 2 \pi \nu_{A}+\alpha_{A} \sin 2 \pi \nu_{A} & \beta_{A} \sin 2 \pi \nu_{A} \\
-\gamma_{A} \sin 2 \pi \nu_{A} & \cos 2 \pi \nu_{A}-\alpha_{A} \sin 2 \pi \nu_{A}
\end{array}\right)
\end{aligned}
$$

is the full-turn transfer matrix for the horizontal plane of the normal modes, and $B$ is written similarly in the vertical plane. $T, U$, and $V$ are $4 \times 4$ matrices. $A, B$, and $C$ are $2 \times 2$ matrices. $I$ is the $2 \times 2$ identity matrix. 
In PLS-II, $\theta_{x}$ is mainly caused by nonsimilarity of the bump magnets, and $\theta_{y}$ is mainly caused by the leakage field in the septum magnet. However, a large horizontal oscillation by $\theta_{x}$ can also cause a vertical oscillation through the coupling element in Eq. (1). The vertical kick strength caused by the leakage field can be converted from the integrated leakage field [Fig. 1(c)] as $\theta_{y}[\mathrm{rad}]=0.29979 \times$ $\mathrm{Bl}[\mathrm{T} \cdot \mathrm{m}] / \mathrm{E}[\mathrm{GeV}]$ [7]. While the injection bump is being constructed, the stored beam's position changes along the $x$ axis [Fig. 1(c)]. Fitting the data linearly in the figure and using the relation between the field integral and the kick yield the relationship between the kick strength and bump height:

$$
\theta_{y}[\mathrm{rad}]=-0.00147 \times h[\mathrm{~m}],
$$

where $h$ is the bump's height in the injection region. Bump magnets $K_{1}, K_{2}, K_{3}$, and $K_{4}$ are symmetrically located on both sides of the injection point, and no optical elements (e.g., quadrupole magnet, sextupole magnet) are placed among the bump magnets in PLS-II, so the bump height can be simplified to [8]

$$
h[\mathrm{~m}]=-\theta_{k}(t)[\mathrm{rad}] \times L_{12}[\mathrm{~m}],
$$

where $\theta_{k}$ is the deflection angle by the bump magnet and $L_{12}=1590 \mathrm{~mm}$ is the center-to-center distance between $K_{1}$ and $K_{2}$. The bump height is proportional to the bump magnetic field in Eq. (6). The bump magnet is operated at a $10-\mathrm{Hz}$ repetition rate and has a $7-\mu$ s half-sine wave. The kick to construct the injection bump can be formulated as

$$
\theta_{k}(t)[\mathrm{rad}]=\theta_{0}[\mathrm{rad}] \sin \left(\pi \times \frac{t}{T_{k}}\right),
$$

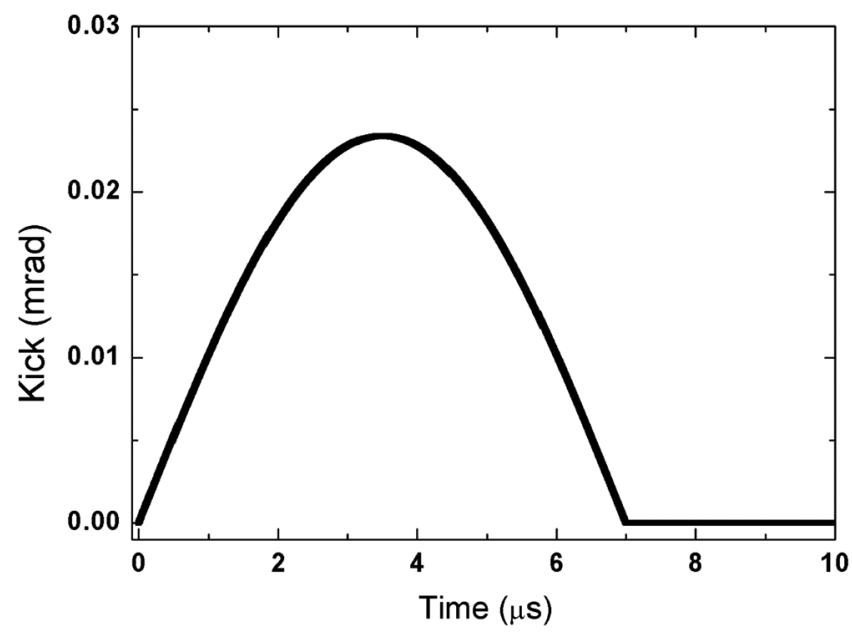

FIG. 2. Kick caused by the leakage field from the septum magnet. This kick is generated due to the change in the septum leakage field along the horizontal injection bump. Therefore, the shape of the kick depends on the field profile of the field of the bump magnet. where $\theta_{0}=10 \mathrm{mrad}$ is the maximum deflection angle of the bump magnet, $T_{k}=7 \mu \mathrm{s}$ is the pulse length of the bump magnet, and $\theta_{k}=0$ after $7 \mu$ s (the bump magnet is off). Substituting Eqs. (6) and (7) into Eq. (5) yields the formula to represent the kick by the leakage field while the bump magnet is on:

$\theta_{y}(t)[\mathrm{rad}]=0.00147 \times \theta_{0}[\mathrm{rad}] L_{12}[\mathrm{~m}] \sin \left(\pi \times \frac{t}{T_{k}}\right)$.

Equation (8) yields the kick caused by the leakage field from the septum magnet. By solving Eq. (1) for every turn, we can estimate the stored-beam oscillation during injection. The leakage field from the septum magnet causes a kick (Fig. 2). $\theta_{x}$ can also be considered straightforwardly according to error sources, and $\theta_{x}=\theta_{y}=0$ when the bump magnet is off (after $7 \mu \mathrm{s}$ ) in Eq. (1).

The stored-beam oscillation by a kick can be illustrated schematically using a normalized phase space [Fig. 3(a)]. The normalized coordinates are defined as [9]

$$
Y=\frac{y}{\sqrt{\beta}}, \quad P=\frac{\alpha y+\beta y^{\prime}}{\sqrt{\beta}},
$$

where $y$ and $y^{\prime}$ are the vertical position and the angle of the beam in Eq. (1). The stored-beam motion by the kick of the leakage field from the septum magnet is expressed in normalized coordinates as

$$
\begin{aligned}
& Y_{n+1}=Y_{n}, \\
& P_{n+1}=P_{n}+\sqrt{\beta} \theta_{n},
\end{aligned}
$$

where $\beta$ is the Twiss parameter at the septum magnet and the kick $\theta$ is 0 after the seventh turn. The stored beam has the first kick at $(X=0, P=0)$ and moves along the circle in the clockwise direction [dotted line in Fig. 3(a)]. In the next turn, the stored beam has the second kick after a $54^{\circ}$ phase advance (the vertical fractional tune is 0.15) and moves along a new circle. The stored beam consecutively receives a kick at every turn up to the seventh and afterward travels along the same circle without a kick (black line in Fig. 3). The suppression scheme by the fast counterkicker can be also described conceptually [Fig. 3(b)]. For simplicity, we consider the same field profile with a kick error shown in Fig. 2. This consideration is necessary when a bunch-by-bunch effect (different kick along the bunch due to a time difference) is considered. Here the amplitude and phase of the fast counterkicker are parameters that can be tuned to minimize residual oscillation. The kicks from the fast counterkicker are assigned to beam oscillation by the kick from the septum leakage field in normalized phase space. Because of different polarity, the fast counterkicker increases the beam oscillation slightly (kick-outward circle) until the second kick but then starts to suppress the beam oscillation (kick-inward circle) at the third turn. Here the effectiveness of the suppression is greatest at the phase of 

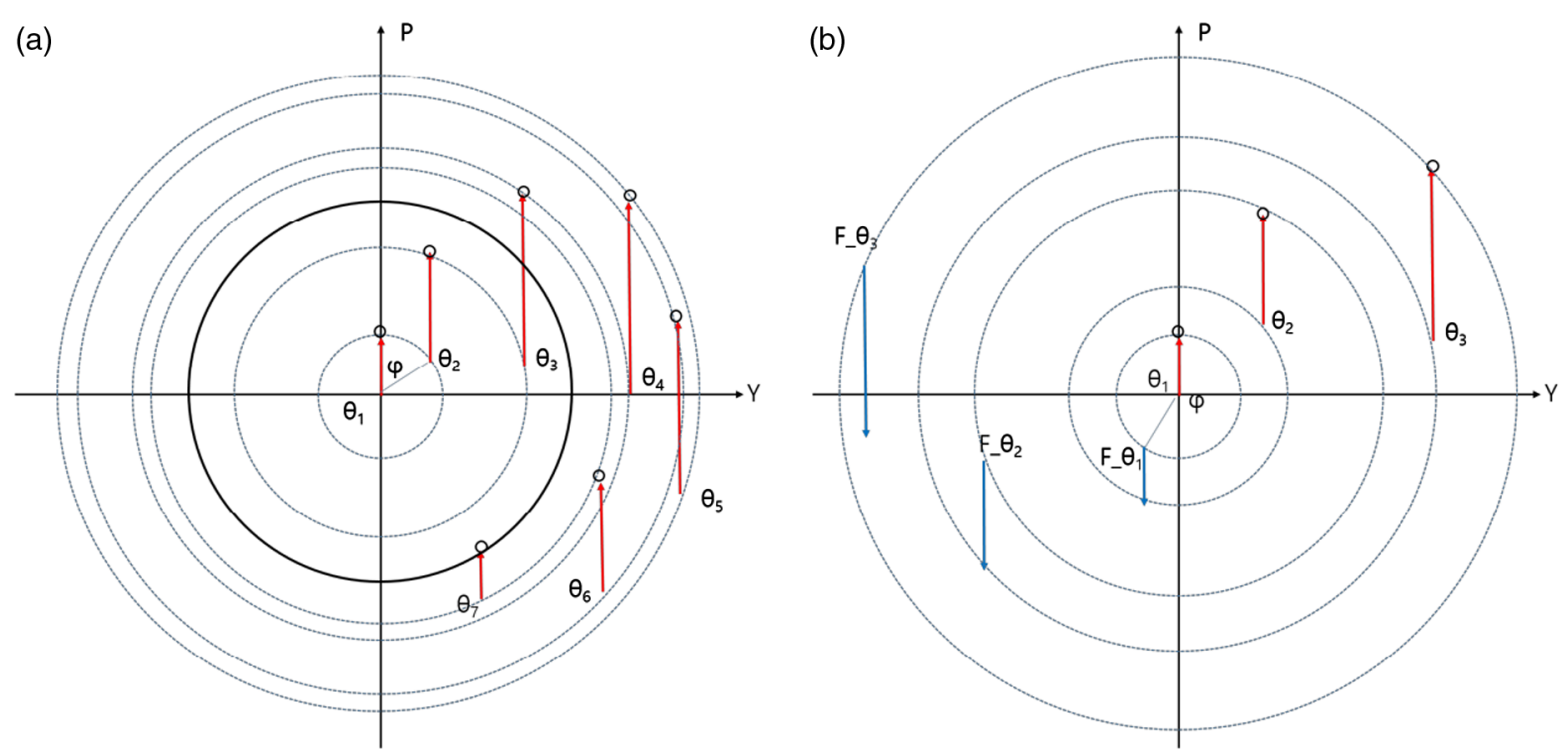

FIG. 3. Stored-beam motion in normalized coordinates. (a) Schematics of the beam oscillation by the leakage field from the septum magnet. $\theta$ is the kick by the leakage field; the subscript is the turn number; $\varphi$ is the phase advance after one turn. (b) Schematics of the beam oscillation by the leakage field and fast counterkicker. Here $\varphi$ is the phase advance between the septum magnet and fast counterkicker. $\mathrm{F}_{\theta}$ is the kick by the fast counterkicker. Each dot represents the motion of the centroid of 100000 particles along turns.

$Y=0$, and the final kick at the seventh turn must be finished at $Y=0$. Therefore, from the third to seventh turns, the fast counterkicker contributes to the suppression of stored-beam oscillation [kick-inside circle of the storedbeam excursion in Fig. 3(b)].

The ELEGANT particle tracking code [10] was used to numerically demonstrate the effectiveness of the fast counterkicker. In the simulation, the determined kick (Fig. 2) was implemented to represent the effect of the septum leakage field. The nonlinear effect is also included, and the betatron tune in the simulation is corrected to operation values $(15.375,9.15)$. The kick by the septum leakage field induced an oscillation in the stored beam [Fig. 4(a)]. To be easily compared with the conceptual description [Fig. 3(a)], the stored-beam oscillation is described in phase space at the location of the septum magnet. The stored beam received kicks at each turn and moved similarly to the case in Fig. 3(a) while the bump magnet was on (up to seven turns). Then the stored beam undergoes betatron oscillation with $100-\mu \mathrm{m}$ amplitude after the bump magnet is turned off. The simulation considered the same waveform in Fig. 2 with a variable kick strength and time delay for the fast counterkicker. The kick strength and time delay were optimized to minimize residual oscillation. The fast counterkicker suppressed stored-beam oscillation due to the septum leakage field [Fig. 4(b)]. The 1 st, 200th, and 400th of 470 bunches are considered in the simulation. Although the fast counterkicker suppressed each bunch differently along the bunch train, the storedbeam oscillations were suppressed to $<10 \mu \mathrm{m}$. However, the error effect of the fast counterkicker is negligible due to its small jitters $(0.1 \%$ rms current jitter and $100 \mathrm{ps}$ rms time jitter). Because this method is simple (just two parameters to tune for operation) and does not require any changes to the beam parameters, the use of a counterkicker to suppress beam oscillation by the leakage field is suggested.

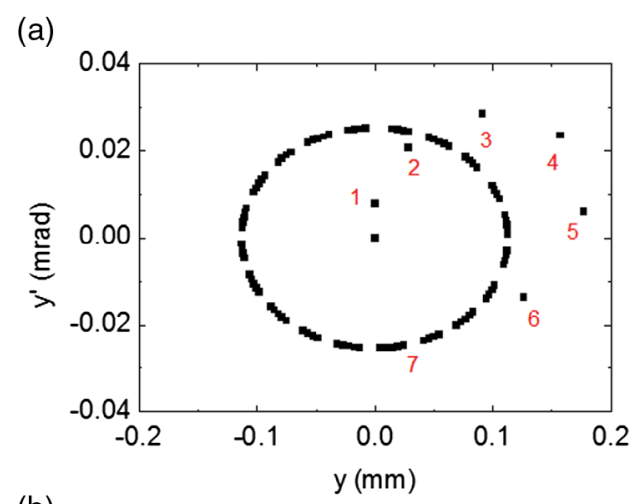

(b)

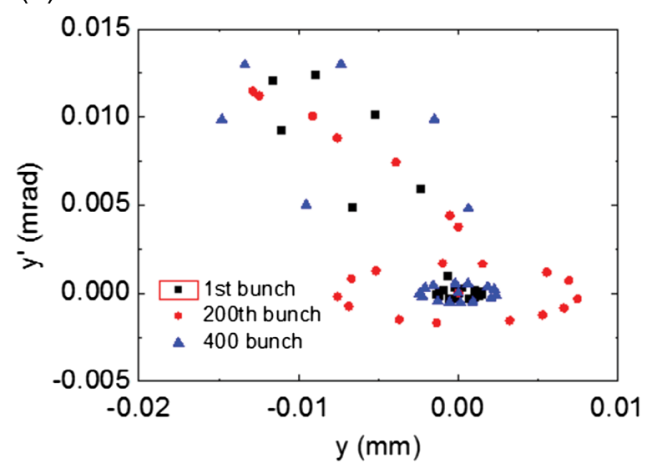

FIG. 4. Stored-beam motion in phase space at the location of the septum magnet. (a) Oscillation due to the septum leakage field. (b) Oscillation suppression by the counterkicker. 


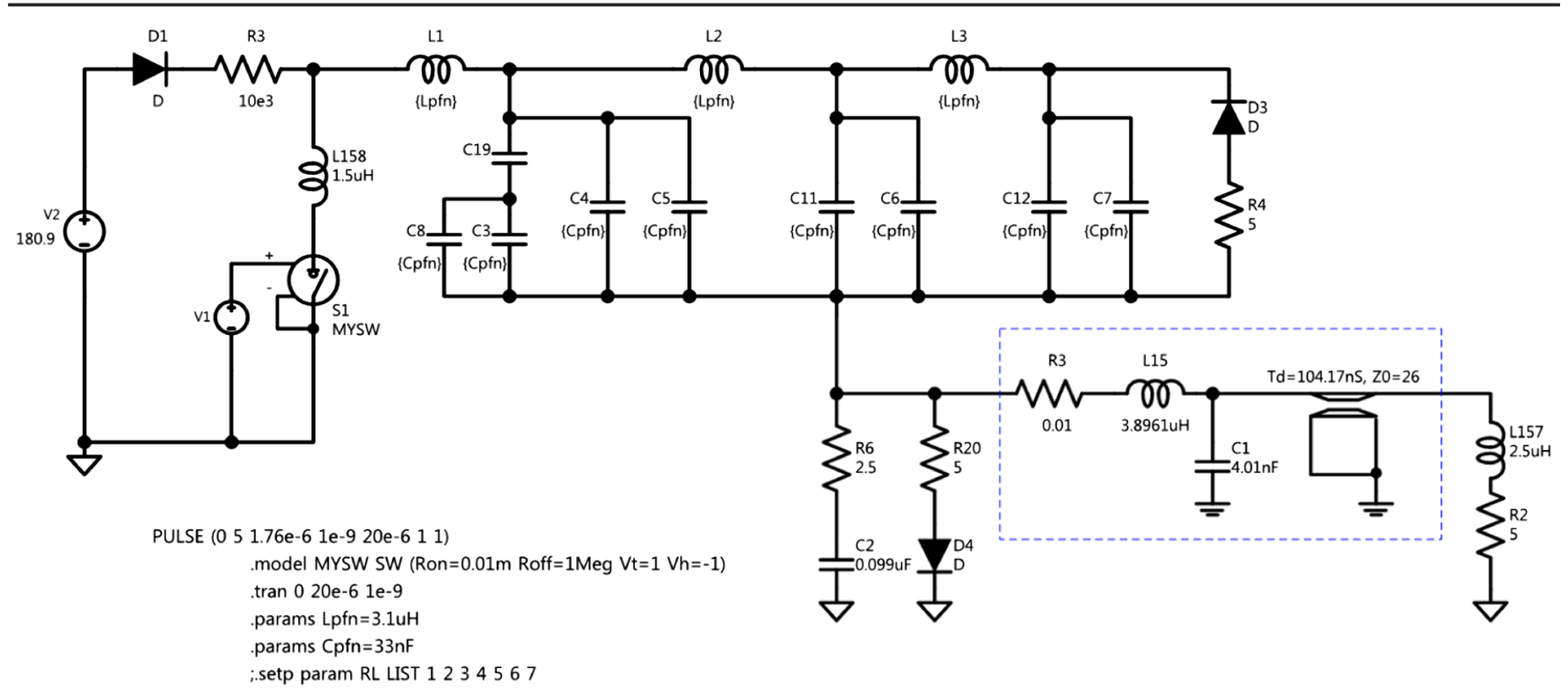

FIG. 5. Circuit diagram for the electric circuit simulation.

\section{FAST COUNTERKICKER SYSTEM}

The fast counterkicker as a compensator to suppress stored-beam oscillation in the horizontal plane was first developed in SPring-8 [11,12]. Efforts to use a fast counterkicker to suppress stored-beam oscillation were conducted in two stages. (1) A correction by the primary fast counterkicker was applied during the user time to suppress a spikelike oscillation that was caused by the dissimilarity of the rising parts of waveform fields in the bump magnets. (2) Following the success of stage 1, a secondary counterkicker was introduced to suppress the broad-width and small-amplitude oscillation that was caused by the dissimilarity of the falling parts of the waveform fields in the bump magnets. The suppression of the photon beam oscillation by the fast counterkicker system was also measured and demonstrated using a turnby-turn beam profile monitor.

The fast counterkicker had been also developed to suppress vertical stored-beam oscillation caused by the Lambertson septum magnet in PLS-II. The ELEGANT simulation suggested that the maximum required kick angle of the fast counterkicker is $30 \mu \mathrm{rad}$ with a pulse width of $7 \mu \mathrm{s}$. The overall fast counterkicker system consists of a dipole air-core magnet, a compact driving power supply circuit that uses $L C$ resonance, and an external high-voltage power supply. The dipole air-core magnet is a ceramic chamber wound with coils to generate both horizontal and vertical magnetic fields. To maintain low impedance for the high stored-beam current, the inner surface of the ceramic chamber is coated with Ti-Mo of $6 \mu \mathrm{m}$ thickness in the horizontal plane and $0.5 \mu \mathrm{m}$ thickness in the vertical plane.

Circuit diagrams (Fig. 5) were developed for a simulation of the electric circuit, and the power supply was constructed (Fig. 6). The power supply (Table I) of the fast counterkicker system is composed of series resonant circuits. The output current waveform produced by this system is a half sinusoid. To realize the short rising time, a MOSFET was used as a switching device. Three-layer pulse-forming networks (PFNs) are used to generate a halfsine-wave current shape, and 25-m coaxial cable from the power supply to the magnet is considered as an equivalent circuit. A power supply of the PFN type consists of many inductors and capacitors and is composed of series and parallel circuit combinations. An event-timing system provides a trigger signal synchronized with the beam injection and can also produce an adequate timing delay. When the trigger signal is received by the power supply, the MOSFET semiconductor switch (NYSW in Fig. 5) is on. Because of the characteristic of a resonant current, an inverse voltage is naturally produced during the negative current blocking. The inverse voltage can become as high as the charging voltage. Therefore, a protection circuit was designed for the switch and connected to it in parallel. The

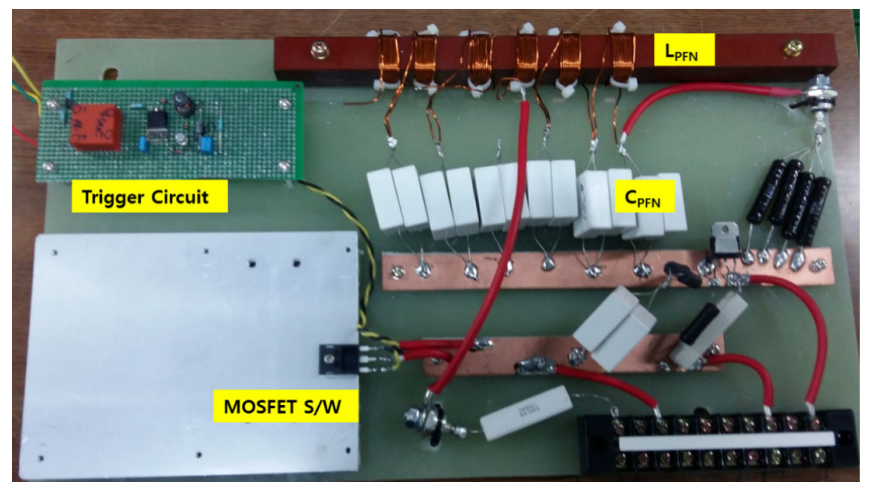

FIG. 6. Photograph of the power supply for the fast counterkicker. 
TABLE I. Specification of the fast counterkicker power supply.

\begin{tabular}{lcc}
\hline \hline Parameter & Value & Unit \\
\hline Range of beam current shake (vertical) & 400 & $\mu \mathrm{m}$ \\
Peak current & 50 & $\mathrm{~A}$ \\
Supply voltage & 500 & $\mathrm{~V}$ \\
Pulse width & 6.8 & $\mu \mathrm{s}$ \\
Total inductance & 5.6 & $\mu \mathrm{H}$ \\
Total capacitance & 2.4 & $\mu \mathrm{F}$ \\
Total impedance & 10 & $\Omega$ \\
Energy per pulse & 0.3 & $\mathrm{~J}$ \\
\hline \hline
\end{tabular}

measured output current (Fig. 7) had a pulse duration of $7 \mu \mathrm{s}$, which is the duration required for the suppression, and the peak current was $10 \mathrm{~A}$ (can be maximized to $60 \mathrm{~A}$ ).

\section{EXPERIMENTAL RESULT}

To suppress vertical stored-beam oscillation while the injection system was operating, the fast counterkicker system has been implemented in sector 7 of the PLS-II storage ring. First, the polarity and trigger timing for beam oscillation by the fast counterkicker system were calibrated. Then the improved oscillation phase and amplitude of the fast counterkicker were scanned to suppress vertical storedbeam oscillation minimally. This procedure to find the optimal setting is simple and straightforward, because tuning requires the adjustment of only the time delay and amplitude. As a result, the stored-beam oscillation was remarkably suppressed (Fig. 8).

Stored-beam motion at the source point for the $2 \mathrm{~A}$ beam line (magnetic spectroscopy beam line) during top-up injections was measured with and without fast counterkicker systems. Phase space beam motion [Fig. 8(a)] was measured indirectly by using beam position monitors

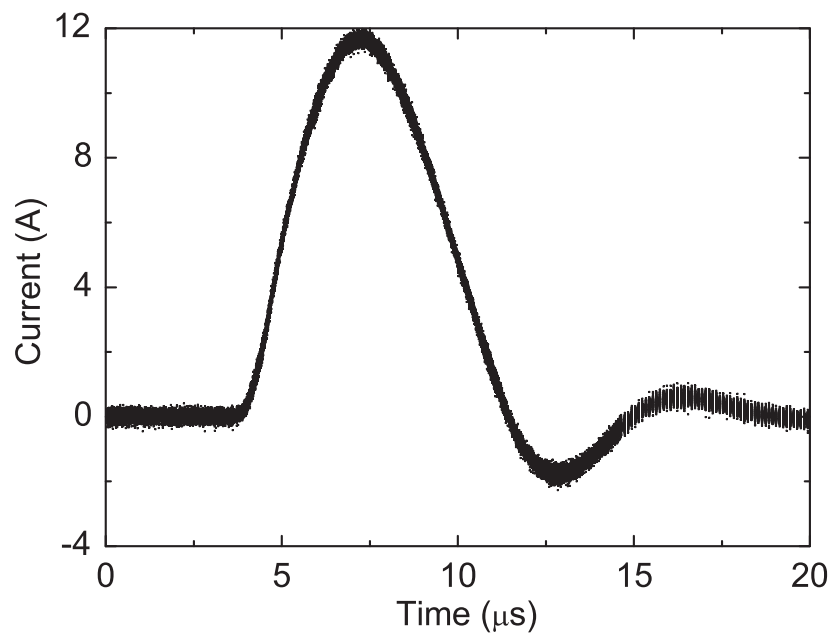

FIG. 7. Measured output current waveform of the fast counterkicker.
(BPMs) upstream and downstream of the 2A insertion device. The large undulator gap in the $2 \mathrm{~A}$ beam line is considered, to ensure the negligible perturbation of the beam trajectory by a field error caused by the insertion device. The stored beam consecutively receives a kick at every turn up to seventh turn and travels along the same circle without a kick after seven turns as described in Fig. 3. The effect of the fast counterkicker was negligible during the first $7 \mu \mathrm{s}$ (kicker waveform duration) [Fig. 8(b)], but stored-beam oscillation was suppressed from 40 to $4 \mu \mathrm{m}$ rms for all BPMs after the kicker system was turned
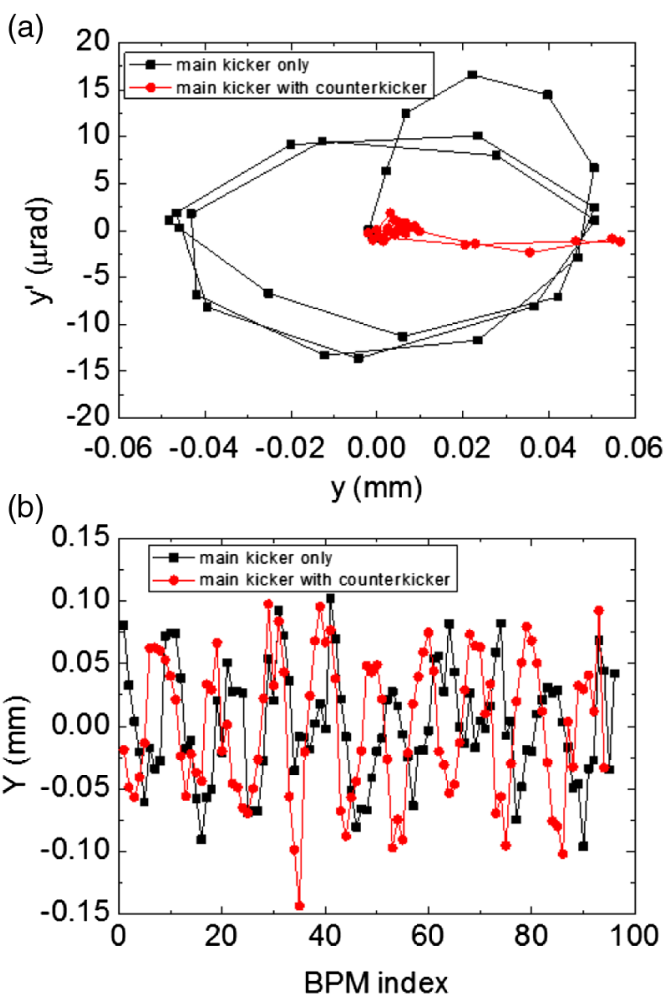

(c)

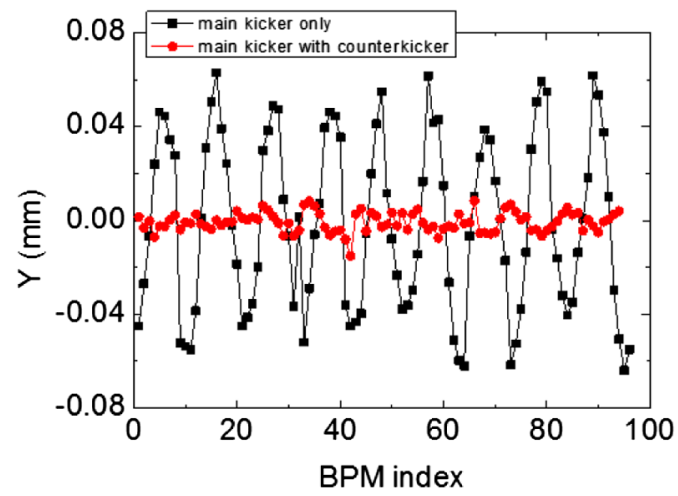

FIG. 8. (a) Measured phase space beam motion at 2A BL (magnetic spectroscopy beam line) with and without a counterkicker. (b) Measured beam oscillation along all BPMs for the fourth turn after the kicker was turned on: oscillation was not suppressed while the kicker was on. (c) Measured beam oscillation along all BPMs after the kicker was off. 

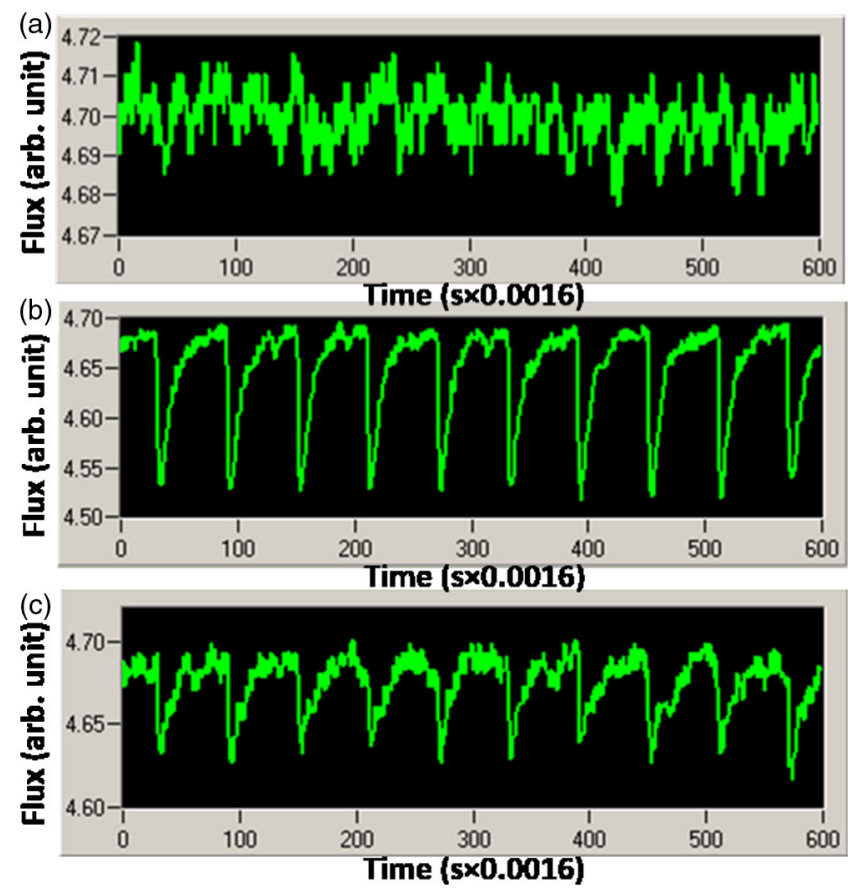

FIG. 9. Measured mesh current at the beam line. Mesh current is acquired at $600 \mathrm{~Hz}$. (a) No injection. (b) Main injection system on. (c) Main injection system and fast counterkicker on.

off [Fig. 8(c)]. The major contributions of the residual $4 \mu \mathrm{m}$ rms oscillation can be explained with the help of Fig. 4(b). Figure 4(b) shows that our fast counterkicker system does not suppress a residual oscillation of less than a few $\mu$ m rms oscillation amplitude when operating with multibunch beams. The optimum suppression required from the fast counterkicker is different for each bunch. In order to further reduce the residual oscillation, a secondary counterkicker system with a shorter pulse duration and a smaller kick amplitude can be considered to suppress the motion of bunches with a relatively larger residual oscillation [e.g., around the 200th bunch of Fig. 4(b)].

The photon flux improvement was also measured at the 2A beam line in PLS-II. The photon source of the beam line is an elliptically polarized undulator with the magnet period of $7.2 \mathrm{~cm}$. The beam flux improvement was measured using a gold mesh just after the exit slit with a vertical gap of $10 \mu \mathrm{m}$. At the exit slit position, the FWHM of the monochromatic central cone was also $\sim 10 \mu \mathrm{m}$, so the mesh current is very sensitive to the variation of beam flux. Data were acquired at a rate of $600 \mathrm{~Hz}$, which is fast enough to monitor the $10-\mathrm{Hz}$ injection transient. The fast counterkicker reduced the dip of the photon beam flux due to injection oscillation by a factor of 3 (Fig. 9).

\section{CONCLUSION}

A fast counterkicker system in PLS-II suppressed the vertical oscillation of a stored beam by a factor of 9 during top-up injection. As a result, the stability of the photon beam flux during top-up injection was improved by a factor of 3 in the magnetic spectroscopy beam line of PLS-II. Horizontal stored-beam oscillation by dissimilarity of magnetic fields in the bump magnets was also suppressed by a fast counterkicker in SPring-8. These results demonstrated that the fast counterkicker system is a useful device to suppress stored-beam oscillation caused by a systematic error and to increase the stability of the beam line flux during top-up injection.

\section{ACKNOWLEDGMENTS}

We thank H. Wiedemann (SLAC) and L. Emery (APS) for useful discussions. This research was supported by the Basic Science Research Program through the National Research Foundation of Korea (NRF2015R1D1A1A01060049).

[1] L. Emery, Recent operational data on continuous top-up operation at the advanced photon source, in Proceedings of the 19th Particle Accelerator Conference, Chicago, IL, 2001 (IEEE, Piscataway, NJ, 2001), p. 2599.

[2] J. Bengtsson et al., Status of the Swiss Light Source project SLS, in Proceedings of EPAC96 (1996), p. 685.

[3] S. Shin et al., Commissioning of the PLS-II, J. Instrum. 8, P01019 (2013).

[4] I. Hwang et al., Top-up operation at Pohang Light SourceII, Rev. Sci. Instrum. 85, 055113 (2014).

[5] D-E. Kim, K-H. Park, H-S. Seo, I. Hwang, S. Shin, K-R. Kim, J-Y. Huang, and S. Nam, Impact of the Lambertson septum magnet on the photon beam flux in the PLS-II beam line experiment, J. Korean Phys. Soc. 64, 197 (2014).

[6] P. Bagley et al., Correction of transverse coupling in a storage ring, in Proceedings of the 1989 Particle Accelerator Conference, Chicago, IL (IEEE, New York, 1989), p. 874.

[7] H. Wiedemann, Particle Accelerator Physics (Springer, Berlin, 2007).

[8] K. Fan, X. Wang, and Y. Pei, A proposed injection system for HLS, Nucl. Instrum. Methods Phys. Res., Sect. A 450, 573 (2000).

[9] H. Takaki and N. Nakamura, Beam injection with a pulsed sextupole magnet in an electron storage ring, Phys. Rev. ST Accel. Beams 13, 020705 (2010).

[10] M. Borland, Advanced Photon Source Report No. LS-278, 2000.

[11] C. Mitsuda et al., Suppression of horizontal beam oscillation by fast kicker magnet system in Spring-8 storage ring, in Proceedings of the International Particle Accelerator Conference, Kyoto, Japan (ICR, Kyoto, 2010), p. 2552.

[12] C. Mitsuda, K. Fukami, K. Kobayashi, M. Masaki, H. Ohkuma, S. Sasaki, K. Soutome, and T. Nakanishi, Suppression of stored beam oscillation at injection by fast kicker in the Spring-8 storage ring, in Proceedings of IPAC14, Dresden, Germany, 2014 (2014), p. 280. 